\title{
Some Aspects of Energy Balance and Tsunami Generation by Earthquakes and Landslides
}

\author{
L. J. RUFF ${ }^{1}$
}

\begin{abstract}
Tsunamis are generated by displacement or motion of large volumes of water. While there are several documented cases of tsunami generation by volcanic eruptions and landslides, most observed tsunamis are attributed to earthquakes. Kinematic models of tsunami generation by earthquakes - where specified fault size and slip determine seafloor and sea-surface vertical motion - quantitatively explain farfield tsunami wave records. On the other hand, submarine landslides in subduction zones and other tectonic settings can generate large tsunamis that are hazardous along near-source coasts. Furthermore, the ongoing exploration of the oceans has found evidence for large paleo-landslides in many places, not just subduction zones. Thus, we want to know the relative contribution of faulting and landslides to tsunami generation. For earthquakes, only a small fraction of the minimum earthquake energy (less than $1 \%$ for typical parameter choices for shallow underthrusting earthquakes) can be converted into tsunami wave energy; yet, this is enough energy to generate terrible tsunamis. For submarine landslides, tsunami wave generation and landslide motion interact in a dynamic coupling. The dynamic problem of a 2-D translational slider block on a constant-angle slope can be solved using a Green's function approach for the wave transients. The key result is that the largest waves are generated when the ratio of initial water depth above the block to downslope vertical drop of the block $H_{0} / W \sin \delta$ is less than 1 . The conversion factor of gravitational energy into tsunami wave energy varies from $0 \%$ for a slow-velocity slide in deep water, to about $50 \%$ for a fast-velocity slide in shallow water and a motion abruptly truncated. To compare maximum tsunami wave amplitudes in the source region, great earthquakes produce amplitudes of a few meters at a wavelength fixed by the fault width of $100 \mathrm{~km}$ or so. For submarine landslides, tsunami wave heights - as measured by $b$, block height — are small for most of the parameter regime. However, for low initial dynamic friction and values of $H_{0} / W \sin \delta$ less than 1 , tsunami wave heights in the downslope and upslope directions reach $b$ and $b / 4$, respectively. Wavelengths of these large waves scale with block width. For significant submarine slides, the value of $b$ can range from meters up to the kilometer scale. Thus, the extreme case of efficient tsunami generation by landslides produces dramatic hazards scenarios.
\end{abstract}

\section{Introduction}

Tsunamis are caused by a rapid displacement of a large water volume. Earthquakes are the most significant source of large tsunamis. Great underthrusting earthquakes in subduction zones uplift the ocean above the fault area by a meter or more, the disturbed ocean surface then propagates away as gravity waves. The largest underthrust earthquakes generate tsunami waves that can cause devastation on both

${ }^{1}$ Department of Geological Sciences, University of Michigan, Ann Arbor, MI, U.S.A. 
local and remote coasts. Other sources of tsunamis are impacts, volcanic eruptions, and landslides.

For a violent volcanic eruption to cause a tsunami, it must either explode underwater or cause debris to fall into the water. This happenstance is rather rare, but one well-known example is the Krakatau eruption in 1883 (NomAnBHoY and SATAKE, 1995). Fortunately, large impacts are even rarer, though potentially very devastating (e.g., WARD, 2000). Landslides are acknowledged to be a ubiquitous and costly natural hazard, but their dynamic energy release is usually small compared to large earthquakes. There are a few documented cases of large subaerial landslides that fell into water and caused large localized waves. One of the most spectacular examples was the Lituya Bay landslide triggered by shaking from the large strike-slip earthquake in southeastern Alaska on July 10, 1958 (Miller, 1960). The landslide fell into a narrow fjord and caused a "slosh" wave that reached a height of $520 \mathrm{~m}$, then continued out the fjord where it was at a height of 10 meters as it entered the ocean. The wave amplitude quickly diminished as it spread out in the open ocean. This indicates that localized tsunamis can be extremely hazardous along a limited coastal extent, yet the total displaced water volume is too small to cause destructive trans-Pacific tsunami waves.

Submarine landslides are mostly hidden from our view. Studies of marine sedimentation show that submarine landslides are a common process and the style ranges from translational block sliding to fluid-like flows (HAMPTON et al., 1996). Hereafter, this wide range of mass wasting is referred to as "landslides"; and, the later quantitative modeling will be restricted to translational block sliding. Despite the overall geologic fact that submarine slides must be common, it is rare to directly observe any slide event. The most famous submarine slide was the Grand Banks slide of November 18, 1929, which was documented by the breaking of trans-Atlantic cables that crossed the slide area. Later studies showed that the peak slide velocity was about $3 \mathrm{~m} / \mathrm{s}$, and that the volume of material is about 1 to $5 \times 10^{11} \mathrm{~m}^{3}$ (PIPER et al., 1985; HASEgAWA and KANAMORI, 1987). This submarine slide raised a regional tsunami that was destructive along the Burin Peninsula of Canada.

Some earthquakes cause tsunamis that are unusually large relative to the seismic waves (KANAMORI, 1972; ABE, 1979). Several explanations have been proposed for these "tsunami earthquakes," including an unusual earthquake rupture process and triggered submarine landslides. One recent case of triggered subaerial/submarine slides is the 1992 Flores Island event where it appears that the landslides caused a local "enhancement" of tsunami heights (HIDAYAT et al., 1995; IMAMURA et al., 1995). Another recent example where a submarine slide may have caused a large localized tsunami is the 1998 Papua New Guinea event (KAwATA et al., 1999; TANiOKA, 1999; TAPPIN et al., 2003; SweEt and Silver, 2003). As more detailed teleseismic and regional quantitative tsunami wave modeling is combined with marine geophysical surveys, more cases of triggered-landslide tsunamis may be 
exposed. These examples now compel us to develop both generalized and detailed models of tsunami wave generation by landslides.

To determine whether large localized tsunami waves are caused by earthquake faulting or by a submarine slide, we need to develop a simple yet useful way to compare the generation of tsunami waves by earthquakes and landslides (MURTY, 2003). To zeroth order, larger tsunamis are generated by larger earthquakes and larger landslides. Of course, wave propagation effects away from the source region are also important for final runup heights (see WARD, 2000 for review and references). We know that seismic moment is the best overall measure of earthquake size, and seismic moment combined with fault size and orientation quickly give us the displaced water volume. For landslides, it would seem that total material volume, or total distance traveled, or some combination of these two parameters would be the best measures of landslide size. But how does this size translate into a predictable tsunami wave height? An experimental approach can determine some empirical rules for specific parameter choices of landslides (e.g., WATTS, 1998), but it would be difficult to test the parameter range relevant to the geologic spectrum of slides (these issues are discussed in WATTS and BORRERO, 2003). The main contribution of this paper is to answer the above question with the simplest possible dynamic model of a submarine landslide.

For earthquake generation of tsunami waves, purely kinematic models specify that ocean surface vertical deformation mirrors the solid surface vertical deformation due to static earthquake faulting (Fig. 1). Some seismologists have extended this kinematic approach to landslide generation, but the fundamental problem is

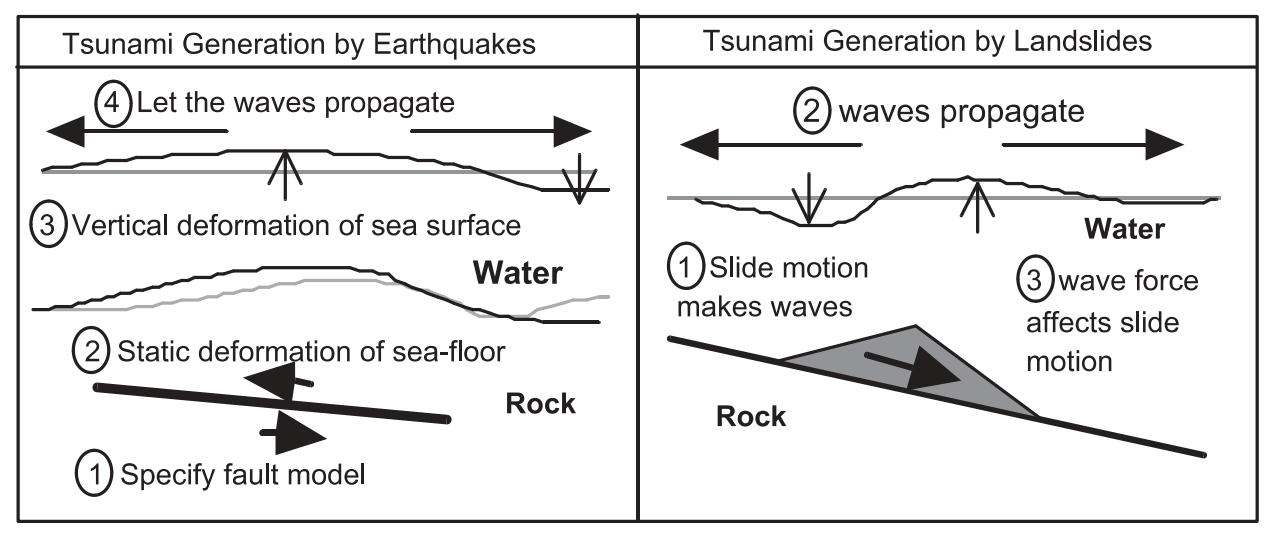

Figure 1

Schematic introduction to tsunami generation by earthquakes and submarine landslides. On the left, the kinematic modeling scheme for earthquake faulting is depicted. On the right, the interaction between wave generation and wave resistance force on the slider is depicted. In general, we must solve the full dynamic problem to generate waves from a block sliding due to its gravitational force; though, strong interaction only occurs in a limited part of the parameter regime. 
that an ad hoc specification of slide velocity will be dynamically incorrect. At the other extreme of the modeling spectrum are fully coupled and dynamic nonlinear numerical models of slide and wave interaction and propagation (e.g., SYNOLAKIS and Tadepalli, 1993; Titov and Synolakis, 1993; Johnson and Mader, 1994; GrILli and WATTS, 1999). Here, we find a useful intermediate approach where the slide velocity is determined by the landslide dynamics and the tsunami wave generation interacts with landslide dynamics, yet the approximations allow for an "almost-analytic" solution that yields general statements on the basic principles of tsunami generation by landslides. Another key question is how to compare the size of earthquakes and landslides. Energy release is probably the best relative measure of earthquake and landslide size. The efficiency of energy conversion into tsunami waves is then a key parameter. We will also estimate maximum wave amplitude at the source for both earthquakes and landslides; this alternative measure of tsunami size may be more useful for modeling and hazards assessment.

\section{Energy Balance and Tsunami Generation by Earthquakes}

Kinematic models for tsunami generation by earthquakes are well developed and accepted (OKAL, 1988; SATAKe, 1995). Consequently, the conversion of energy from earthquake faulting into tsunami waves has received little attention. In order to compare earthquakes and landslides based on energy, we will calculate the efficiency of tsunami wave generation by earthquakes. First we review energy basics and then show that only a small fraction of earthquake energy goes into tsunami waves.

\subsection{Energy Balance for Earthquakes}

Energy balance is a fundamental consideration in the physics of seismic sources and waves. Since earthquakes reduce the shear stress in some rock volume around the fault, stored elastic strain energy is released and deposited into two main reservoirs: waves and "friction" in the faulted region. Shortly after the advent of seismic source theory, HASKELL (1964) and others showed that the internal strain energy change associated with the shear dislocation earthquake model is given as: $\Delta E=\tau_{a v} D A$, where $D$ is the fault slip averaged over fault area $A$, and $\tau_{a v}$ is the average shear stress as the fault slips. This formula can be rewritten in terms of the static stress drop of the earthquake:

$$
\Delta E=A\left[\Delta \sigma D / 2+\tau_{\text {final }} D\right],
$$

where $\Delta \sigma$ is the stress drop, and $\tau_{\text {final }}$ is the shear stress on the fault after the earthquake. Note that gravitational energy changes do not appear in Equation (2.1). As shown by DAHLEN (1977), the earthquake-induced changes in rotational, 
gravitational, and hydrostatic energies can be much larger than the earthquake energy in Equation (2.1), nonetheless all of these large first-order energy changes are perfectly balanced so that the net energy available for fault friction and seismic waves is precisely the same as given by ignoring gravitational forces and the hydrostatic stress state.

Although seismologists can estimate $\Delta \sigma, D$ and $A$ from various observations, the level of absolute stress (i.e., $\tau_{\text {final }}$ ) is still a matter of great speculation and opinions. Indirect estimates based on the accessory observations of heat flow suggest that $\tau_{\text {final }}$ is not too much larger than $\Delta \sigma$ in strike-slip settings, but may be ten times larger than $\Delta \sigma$ in subduction zones (e.g., RUFF, 1999). Given this uncertainty in absolute stress level, it is reasonable to use the lower bound on earthquake energy, which is based on observed quantities:

$$
\Delta E_{\min }=\Delta \sigma D A / 2 .
$$

The next problem is how the shear energy release in Equation (2.2) is partitioned between fault friction and seismic waves. The partitioning is determined by the spacetime history of dynamic friction during faulting, and careful seismic wave studies are required to quantify this partitioning (e.g., KIKUCHI and FUKAO, 1988; WINSLOW and RUFF, 1999). Here, we ignore this partitioning problem and use $\Delta E_{\min }$ as a measure of overall earthquake energy. Recall that seismic moment is defined as: $M_{o}=\mu D A$, where $\mu$ is source region shear modulus. Hence we can rewrite $\Delta E_{\min }$ as:

$$
\Delta E_{\min }=\Delta \sigma D A / 2=\Delta \sigma M_{o} / 2 \mu .
$$

Seismic moment is an earthquake parameter that can be reliably and systematically determined. Let us accept one of the key maxims of earthquake systematics that all large shallow earthquakes have the same stress drop of about $3 \mathrm{MPa}$. Since a crustal shear modulus is about $30 \mathrm{GPa}$ for all shallow tectonic environments around the world, we can evaluate Equation (2.3) into the following universal formula that immediately gives an energy estimate once seismic moment is known (KANAMORI, 1977; VASSILIOU and KANAMORI, 1982):

$$
\Delta E_{\min }=5 \times 10^{-5} M_{o} .
$$

To give some numbers for great underthrust earthquakes, let a fault zone extend for $100 \mathrm{~km}$ in the downdip direction and $200 \mathrm{~km}$ along strike, and let the slip be 10 meters. The seismic moment is $6 \times 10^{21} \mathrm{Nm}$, which translates into a moment magnitude $M_{w}$ of 8.5 . We can estimate the earthquake energy by multiplying this moment by the universal factor in Equation (2.4) to obtain the value of $3 \times 10^{17}$ Joules. The largest earthquake in the 20th century (1960, Chile) has an energy estimate of about $10^{19} \mathrm{~J}$. There were about ten underthrusting earthquakes with $M_{w} \geq 8.5$ in the 20th century. 


\subsection{Tsunami Generation by Earthquakes}

Large shallow earthquakes that occur beneath the ocean can cause tsunamis that bring destruction to local coastlines, or across the ocean in some cases. Tsunamis are caused by the fault-induced seafloor deformation displacing large volumes of water (Fig. 1). While this basic principle has been understood for a long time, quantitative modeling of tsunami waveforms is a rather recent advance.

If the ocean is viewed as a uniform global layer, then seismic source theory and normal mode formalism can be extended to include tsunami water waves, which are dominated by wave periods much longer than earthquake duration times (OKAL, 1988; WARD, 2000). However, the strong variations in bathymetry play a dominant role in the propagation of tsunami waves. Thus, an alternative approach is to place a finite fault within an elastic half-space, calculate the static vertical displacements, displace the water up and down to disrupt the ocean surface, then numerical methods propagate the tsunami waves across the detailed bathymetry of the region (e.g., SATAKE, 1995). This methodology provides results consistent with other constraints. The method works because earthquake duration is usually less than one minute, even for great earthquakes, while the fault-induced tsunami waves have significant energy at wave periods more than one minute. This separation of time scales between the seismic source duration and tsunami wave period allows us to complete the seismic source process before we activate the tsunami wave propagation dynamics. While this kinematic approach is now well-accepted, the implications for earthquake energy balance have not been fully considered.

\subsection{Tsunami Energy Balance for Earthquakes}

At first glance, we might think that an ocean layer on top of the solid surface significantly alters the earthquake energy balance because the earthquake must "lift" the entire ocean. However, a more careful look at the energy balance shows that the first-order energy required to "lift the ocean" is balanced by the first-order change in the hydrostatic energy of the ocean-rock column. The details of this energy balance are not detailed here; they follow the same arguments of DAHLEN (1977) for the whole earth problem.

Thus, we only need to be concerned about the second-order energy that is carried away from the source region by the tsunami waves. In the standard kinematic modeling of tsunami waves, the wave energy that propagates away is the potential energy given up by the "hills and holes" in the water surface returning to the reference level state. Since the "hills and holes" are only a few meters high, even for great earthquakes, this amount of potential energy is much less than the energy required to lift the entire ocean layer by a meter. But is this second-order tsunami wave energy comparable to the minimum earthquake energy? We can derive a simple relationship that connects seismic moment to tsunami wave energy for the typical geometry of a great underthrust earthquake. Place the up-dip edge of the 100-km 
wide fault in an elastic half-space at a depth of $10 \mathrm{~km}$ below the traction-free surface, and the dip angle is $20^{\circ}$. Given the shear dislocation of $10 \mathrm{~m}$, the maximum vertical uplift at the surface is $4.7 \mathrm{~m}$ just above the up-dip edge, and the maximum surface subsidence is $1.3 \mathrm{~m}$ about $50 \mathrm{~km}$ behind the down-dip edge of the fault. To give our generic earthquake a finite size, let the along-strike length be $200 \mathrm{~km}$, then we have the same moment as before $\left(6 \times 10^{21} \mathrm{Nm}\right)$, and recall that the value for $\Delta E_{\min }$ is $3 \times 10^{17} \mathrm{~J}$. After displacing the water surface by the same amount as the vertical ground motion, the gravitational potential energy available for the tsunami waves is $7.1 \times 10^{14} \mathrm{~J}$. As the tsunami waves begin to propagate away from the fault region, this energy becomes equally partitioned between the potential and kinetic energy of the waves. In particular, let the waves propagate in a uniform 4-km thick ocean layer, then we find that the equi-partitioning was achieved to within $1 \%$ after just 11 minutes of wave computation. The total tsunami wave energy is a small fraction of the minimum earthquake energy of $3 \times 10^{17} \mathrm{~J}$ - only about 0.002 of the available energy need be diverted into tsunami wave energy. Given the huge errors in the actual measurement of earthquake energy, loss of this tiny fraction would hardly be noticed. On the other hand, the tsunami waves associated with this small energy fraction can be large and destructive.

Figure 2 shows a simplified diagram of the vertical deformation that can be used to develop a simple algebraic connection from earthquake parameters to tsunami

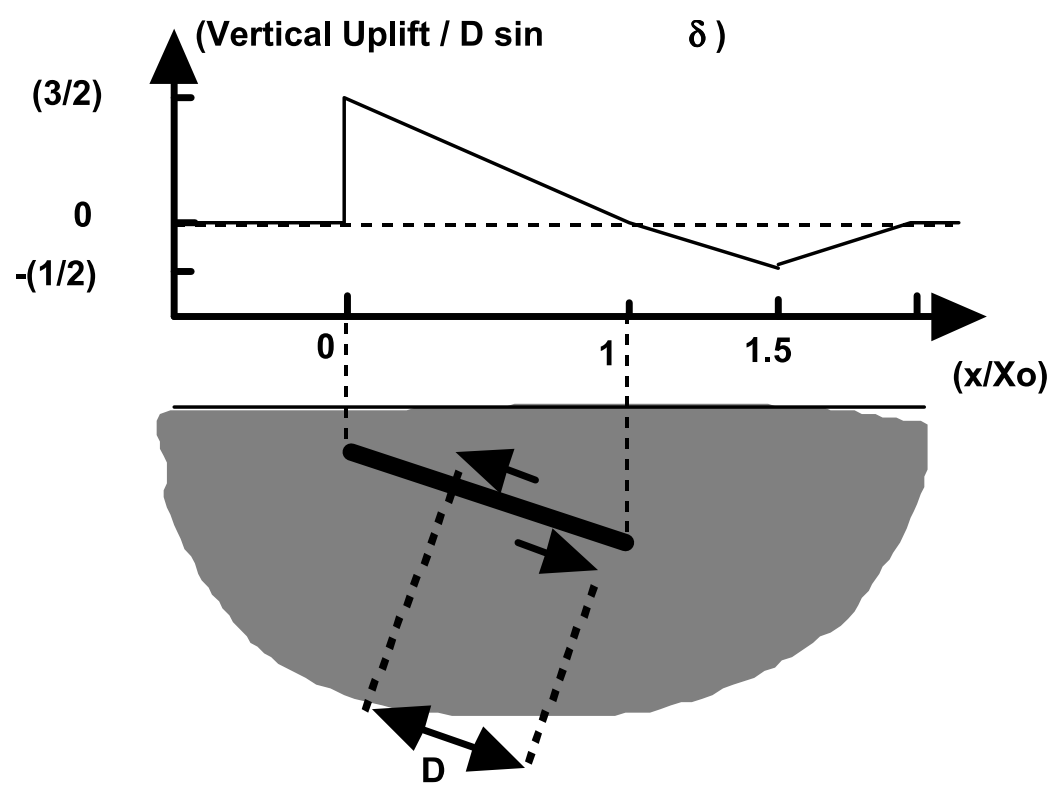

Figure 2

Stylized faulting model and consequent free-surface vertical deformation are shown in this 2-D crosssection view. The simplified surface deformation closely matches the exact results, yet allows for a simple analytical formula for the tsunami wave energy. 
wave energy. We estimate the maximum uplift above the updip edge as: $\Delta V_{\max }(x /$ $\left.X_{o}=0\right)=1.5 D \sin \delta$; and the maximum subsidence behind the fault as $\Delta V_{\min }(x /$ $\left.X_{o}=1.5\right)=-D / 2 \sin \delta$, where $X_{o}$ is the horizontal projection of the fault width, i.e., $X_{o}=W \cos \delta, \delta$ is fault dip angle, and $D$ is the fault shear dislocation. Note that application of the above simplistic description to our above example great earthquake fault yields $\Delta V_{\max }$ of $5.1 \mathrm{~m}$ and $\Delta V_{\min }$ of $-1.7 \mathrm{~m}$, close to the values from the full numerical calculation. The simplistic triangular functions for vertical motion can be integrated along $x$ and fault strike to obtain the following formula for tsunami wave energy: $\Delta E_{\text {tsunami }}=g \rho_{w} W L(5 / 12) \cos \delta(\mathrm{D} \sin \delta)^{2}$, where $\rho_{w}$ is water density and $g$ is gravitational acceleration. Note that this formula contains the product of the fault area and displacement, hence we multiply and divide by the shear modulus $\mu$, to extract the seismic moment of the earthquake:

$$
\Delta E_{\mathrm{tsunami}}=M_{o} g \rho_{w} / \mu(5 / 12) \cos \delta D \sin ^{2} \delta .
$$

To demonstrate that the above simple expression is useful, substitute the particular values for $M_{o}, D$, and $\mu$ for our example great earthquake to obtain: $\Delta E_{\text {tsunami }}=9.1 \times 10^{14} \mathrm{~J}$, just $28 \%$ larger than the value calculated for the exact $3-\mathrm{D}$ faulting model. Accepting the above algebraic formula as a good estimator of tsunami energy, we can rewrite it to look quite similar to the earthquake energy formula. First, let us define the approximate static stress drop as: $\Delta \sigma=\mu D / W$, or $\Delta \sigma / \mu=D / W$, and finally $D=W \Delta \sigma / \mu$. Thus, the energy is:

$$
\Delta E_{\mathrm{tsunami}}=M_{o} \Delta \sigma g \rho_{w} / \mu^{2}(5 / 12) W \cos \delta \sin ^{2} \delta .
$$

Recognizing that $M_{o}(\Delta \sigma / 2 \mu)$ is the minimum earthquake energy $\Delta E_{\min }$, we have for the ratio of tsunami to earthquake energy: $\Delta E_{\text {tsunami }} / \Delta E_{\min }=g \rho_{w} / \mu(5 / 6) W \cos \delta$ $\sin ^{2} \delta$. Notice that $W \sin \delta$ is the depth range of fault width $W$, which provides the "natural" vertical depth scale for the hydrostatic pressure: $g \rho_{w} W \sin \delta$, that is compared to the shear modulus as:

$$
\Delta E_{\text {tsunami }} / \Delta E_{\min }=g \rho_{w} W \sin \delta / \mu(5 / 6) \sin \delta \cos \delta .
$$

Thus, aside from the geometric factor of $5 / 6 \sin \delta \cos \delta$ that is always less than 1 , the ratio of tsunami to earthquake energy is controlled by the ratio of $g \rho_{w} W \sin \delta$ to $\mu$. Given that the maximum depth extent of the seismogenic plate interface in subduction zones is about $40 \mathrm{~km}$ (TICHELAAR and RUFF, 1993), we can let $W \sin \delta$ be $40 \mathrm{~km}$, choose $\mu$ as its typical crustal value of $30 \mathrm{GPa}$, to obtain an upper numerical bound on this ratio as:

$$
\Delta E_{\text {tsunami }} / \Delta E_{\min }=0.013(5 / 6) \sin \delta \cos \delta .
$$

Therefore, this approximate algebraic formulation shows that the tsunami wave energy shall always be less than $1 \%$ of the minimum earthquake energy. This result shows that the interaction of tsunami waves back on the earthquake rupture process 
can be ignored, hence kinematic models of tsunami wave generation by earthquakes are fully justified from an energy perspective.

\section{Energy Balance and Tsunami Generation by Landslides}

Landslides move due to the force of gravity. The energy release of a landslide is thus the difference between net gravitational potential energy before and after the event. The change in strain energy of the solid earth can be ignored to second-order. For submarine slides, it is the difference between rock and water density that gives net displaced mass. As a surficial process, we can construct good estimates of the total mass of material and the center of mass displacement for many cases. Thus, in contrast to earthquake energy, the overall static energy release of landslides might be reliably estimated for both recent and ancient landslides.

There have been many spectacular and hazardous subaerial landslides, although it now seems that many of the largest slides occur under water. Marine geophysical surveys are capable of shedding light on these submarine slides (see reviews by MoOre et al., 1994; HAMPTON et al., 1996). For reference, the energy release of the 1929 Grand Banks slide is about $3 \times 10^{17} \mathrm{~J}$. However Moore et al. (1994) discuss ancient landslides off many margins of the world, and the Nuuanu slide off the Hawaiian Islands has an energy release of about $2 \times 10^{20} \mathrm{~J}$. When we compare this gravitational energy release to the earthquake energy of the 1960 Great Chilean earthquake (about $10^{19} \mathrm{~J}$ ), we see that these ancient landslides are some of the most energetic events on earth.

Where does the landslide energy go? It is partitioned into the two main reservoirs of the ocean and the solid earth (Fig. 3). As for earthquakes, the partitioning within the solid earth reservoir between friction and seismic waves is controlled by dynamic friction during the slide. The ocean reservoir is further divided into "friction" (i.e., hydrodynamic drag and related phenomenon such as acoustic waves) and surface gravity waves - tsunamis. This partitioning is determined by the details of block motion, in particular whether the fastest slide velocity is close to the wave propagation velocity. Unfortunately, we cannot just look at the "before and after" of a submarine landslide and guess how large the tsunami waves were; we must first solve the dynamic problem of landslide motion.

\subsection{Kinematic Versus Dynamic Landslide Models}

Submarine slides are a complex geologic process, but are idealized hereafter as two-dimensional translational slides down a constant-angle slope. A kinematic description simply specifies the time history of block displacement; then, the tsunami waves that are generated by this history have no influence back on the block (e.g., IwASAKI et al., 1996; MA et al., 1999). The extreme specification is to make the slider 


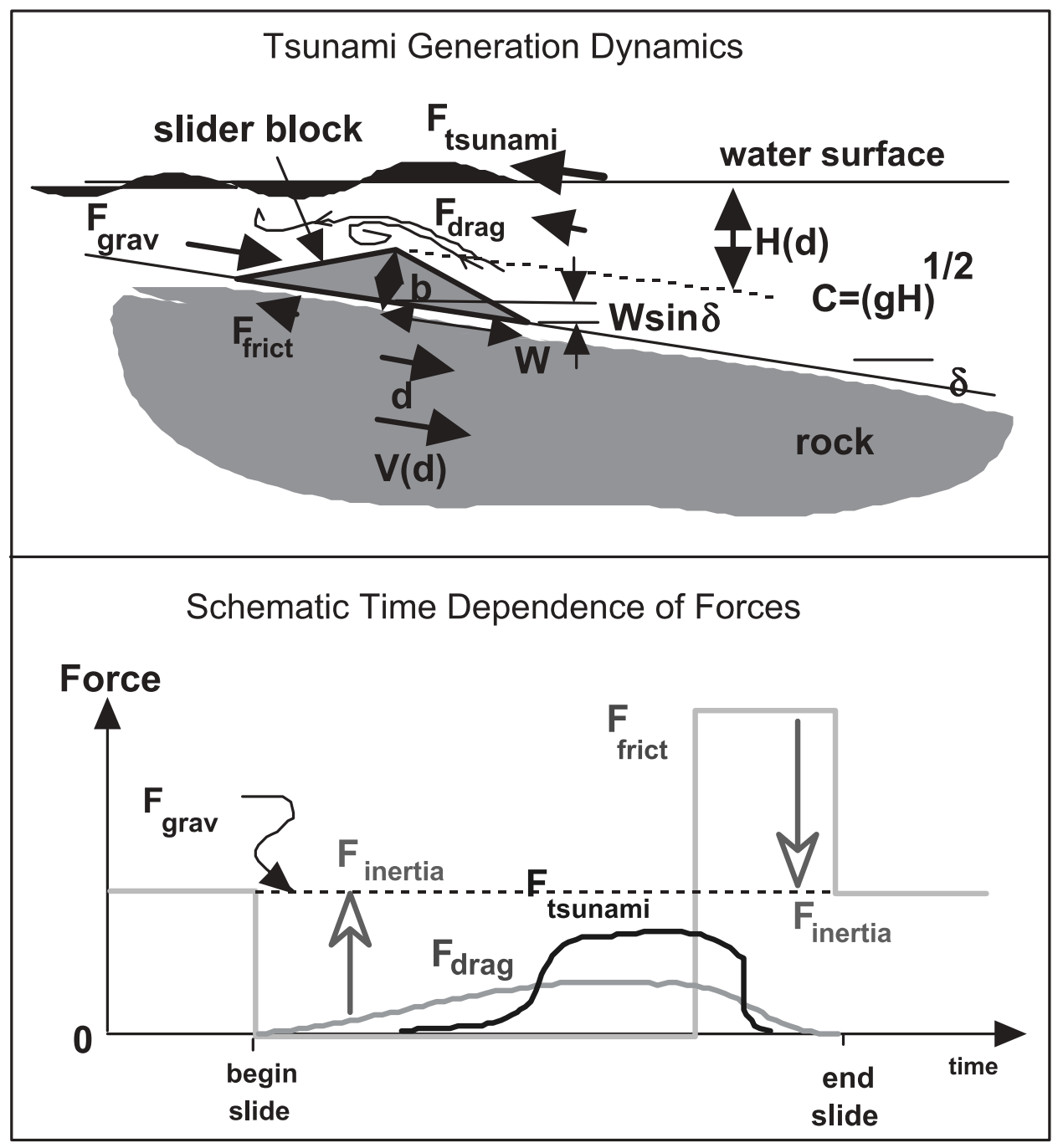

Figure 3

Force balance for the submarine slider block problem. See text for description of all variables. A symmetric triangular slider block was used to produce a simple yet reasonable wave impulse response. The downslope gravitational force is constant throughout the motion, the three resistive forces are interface friction, hydrodynamic drag, and wave resistance. The difference between gravitational force and the sum of resistive forces is the inertia force which thence gives block motion history. A schematic history of a particular slide event is depicted below to suggest the interplay of these force terms.

velocity so fast that it emulates the earthquake generation problem where the source starts and stops before any gravity waves can propagate. While this instantaneous kinematic model is acceptable for the earthquake problem, we cannot accept it for the landslide problem because this ad hoc specification may not conserve energy. To 
experiment with physically plausible kinematic models, one could calculate hundreds or thousands of possible histories - but which one is correct? Perhaps the best approach is to solve the dynamic problem where the velocity of the block and the time-dependent tsunami wave heights are determined in a self-consistent fashion. Since landslides are idealized simply, the mathematical problem reduces down to a single-particle integro-differential equation with nonlinear terms. Although the necessity of solving the dynamic problem greatly complicates tsunami wave generation - it is quite beneficial in the final analysis because the dynamicallyconsistent solutions only traverse a small part of the total kinematic parameter regime. Hence the dynamic solution actually simplifies the scientific problem of tsunami generation by landslides.

\subsection{Dynamics of the 2-D Slider Block}

Figure 3 shows the physical elements in the force balance. The key is that the net gravitational energy given up by the block as it moves down the slope is:

$$
\Delta E_{\text {grav }}=\Delta \rho W b L g d \sin \delta,
$$

where $\Delta \rho$ is the difference between rock and water density, $W$ is half the downslope block width, $b$ is the block height at the center of the symmetric triangular crosssection, $L$ is the along-strike block length, $\delta$ is the slope dip angle, and $d$ is the current block displacement along the slope. The gravitational force acting along the downslope direction is the derivative of Equation (3.1) with respect to $d$ :

$$
F_{\text {grav }}=\Delta \rho W b L g \sin \delta
$$

and it is constant before, during, and after the slide event. The density difference $\Delta \rho$ in Equations (3.1) and (3.2) can be viewed as either the net mass that slides down (good for the static view) or as the net effect of differential hydrostatic pressure acting on the block (better for the dynamic view).

In general, after the slider has stopped with final displacement $d$, the net gravitational energy release in Equation (3.1) is partitioned into a total of four reservoirs: friction along the sliding interface, seismic waves radiated into the solid, frictional drag within the water, and water gravity waves. During the slide, the force balance consists of the following three force types: (i) the constant gravitational force in the downslope direction; (ii) the three resistive forces of sliding interface friction, hydrodynamic drag, and gravity wave generation; and (iii) the inertia force which translates the mismatch between gravitational and resistive forces into block acceleration and deceleration. This force balance along the downslope direction is written as: $F_{\text {inertia }}=F_{\text {grav }}-F_{\text {frict }}-F_{\text {drag }}-F_{\text {tsunami }}$. After dividing the entire equation by the constant $F_{\text {grav }}$, we obtain the following non-dimensionalized force terms:

$$
F_{\text {inertia }} / F_{\text {grav }}=1-F_{\text {frict }} / F_{\text {grav }}-F_{\text {drag }} / F_{\text {grav }}-F_{\text {tsunami }} / F_{\text {grav }},
$$


where the resistive forces are positive when directed upslope, and $F_{\text {inertia }}$ is positive for block acceleration in the downslope direction. As we consider the details of each term, block displacement will be non-dimensionalized by $W$, and velocities will be non-dimensionalized by the "natural" system velocity of $C_{w}=(g W \sin \delta)^{1 / 2}$, where the velocity length-scale is half the downslope vertical drop of the block.

Let us now consider the nature and properties of these forces. We have the fewest physical constraints on the interface dynamic friction force. We know it must drop below the static friction value for the slide to proceed. If the dynamic friction force drops to just slightly less than the static value, then the block slowly creeps down the slope - this will not generate any waves. At the other extreme, friction drops to zero and then the block accelerates as fast as it can, given the other resistive forces. We shall assume this extreme case for the initial phase of slider motion, i.e., $F_{\text {frict }} /$ $F_{\text {grav }}=0$. Eventually, the interface friction term must acquire a value of more than 1 to decelerate and finally arrest the motion of the slider block. Similar to the earthquake partitioning problem, time history of the interface friction force determines both the friction and seismic wave energies.

The hydrodynamic drag force basically depends on water density, slider frontal area, the non-dimensional drag coefficient $D$, and square of slider velocity as follows: $F_{\text {drag }}=D \rho b L V^{2}$. There is a long history of theoretical and experimental development in fluid dynamics over the details of hydrodynamic drag; object shape, surface roughness, and Reynolds number all influence the details of drag. Studies of hydrodynamic drag lead to the discoveries of turbulence and boundary layer separation, but all these details can be lumped into the non-dimensional $D$ - which has a typical value of about 1 , though it can be as low as 0.1 or 0.2 for streamlined bodies at moderately high Reynolds numbers. Given the large length scale of geologic blocks, the overall Reynolds number is very high as soon as the block moves. The dependence of $D$ on $V$ is ignored, but the model will be tested with values of $D$ that cover the range from 0.1 to 1 .

We now consider the tsunami wave force on the block. If a block moves through a water layer with a free surface, then any gravitational surface waves that are generated impart a wave resistance force back on the slider block. There is a long and rich history of work on the wave resistance problem, most of the famous solutions are for "ship waves" (e.g., LAMB, 1932; Lighthill, 1978). LAmb (1932) and Lighthill (1978) also give analytic solutions for the two-dimensional problem of objects moving along the bottom of a uniform depth water layer; or conversely, a water layer flowing over an obstacle. These classic solutions are for the steady-state problem where the object has moved with a constant velocity since $t=-\infty$. We focus on those cases where the dominant wavelength is much larger than the water depth. In these solutions, wave behavior is largely determined by the relationship between object velocity $V$, and wave velocity for long wavelengths, $C=(g H)^{1 / 2}$ where $g$ is gravitational acceleration and $H$ is water layer thickness. When the $V / C$ ratio is much smaller than 1 , then both steady-state wave amplitude and wave resistance force are 
small. As $V / C$ approaches 1, both wave amplitude and resistive force grow and eventually we must abandon the premise of linear long-wave theory. If $V / C$ is much larger than 1, the water layer just rushes over the object and returns to its original state without significant wave production. While these steady-state solutions are instructive, we need to solve the transient problem where the block starts at zero velocity, accelerates to some maximum velocity, and then eventually stops. The transient problem is more difficult than the steady-state problem, yet the classic treatments still provide the basis for developing a Green's function style solution appropriate for the transient problem. WARD (2000) shows how to integrate a particular source contribution for a 3-D tsunami wave generation problem. In comparison, we will use a 2-D Green's function that give waves propagating in the downslope and upslope directions. But for extending these results to a dynamic model, we need to use the current slider velocity to generate a wave, add it to the waves previously generated, and then calculate the wave resistance force that feeds back into the force balance equation that determines the change in slider velocity. There is the additional complication that wave velocity changes as a function of slider displacement, i.e., $C(d)=\left[g\left(H_{o}+d \sin \delta\right)\right]^{1 / 2}$, where $H_{o}$ is initial water depth. Our experience in seismic source theory suggests that the first part of this problem - wave generation and propagation due to an unknown and variable velocity time history - can be written as the convolution between the "impulse" wave generation function of the triangular block and the velocity history function. For the second part of the problem, we simply use the average net wave pressure differences on the front and back faces of the block to calculate the wave resistance force. This entire cycle of wave generation and wave resistance can be written as an analytic convolution integral over a nonlinear function of velocity. The final wave resistance then depends on the sum of two convolution integrals for the downslope and upslope propagating waves. This non-dimensionalized form of the $F_{\text {tsunami }} / F_{\text {grav }}$ ratio can be written:

$$
F_{\text {tsunami }} / F_{\text {grav }}=\rho / \Delta \rho b / W \sin \delta\left[A_{>}+A_{<}\right],
$$

where $\rho / \Delta \rho$ is the non-dimensional density ratio of water to rock-water, $b / W \sin \delta$ is the non-dimensional "scale" for tsunami wave height, and $A>$ and $A<$ are the nondimensional average wave-height force functions above the slider for down- and upslope waves. $A_{>}$and $A_{<}$are calculated from the wave-making functions, $M_{>}$and $M_{<}$, which are given by:

$$
\begin{aligned}
& M_{>}=[+\operatorname{Box}(x ; 0, W)-\operatorname{Box}(x ; W, 2 W)] *\{1 /[C / V-1]\}(x) \\
& M_{<}=[-\operatorname{Box}(x ; 0, W)+\operatorname{Box}(x ; W, 2 W)] *\{1 /[C / V+1]\}(x),
\end{aligned}
$$

where $x$ measures the compressed/extended horizontal distance from the leading edge of the wave back to the slider as $\partial \mathrm{x} / \partial \mathrm{d}=C / V \pm 1$ for the downslope(-)/upslope(+) waves, normalized by $W, \operatorname{Box}(x ; A, B)$ is the "boxcar" function that is 1 within the range from $A$ to $B$, zero elsewhere, $\{1 /[C / V \pm 1]\}(x)$ are the velocity kernel 
functions, and * represents the convolution integral over $x$ from the furthest advance of the initial wave back to the slider.

Before proceeding, let me review the approximations and shortcomings of the above formulas. The slider block shape can be assumed to have a symmetric triangular cross section so that the impulse response for water surface vertical velocity is a positive "boxcar" function above the leading face and a negative "boxcar" function above the trailing face. Also, the velocity kernel function is evaluated at the block apex and water depth is assumed to be small enough that wave dispersion effects at the "boxcar" wavelength can be ignored. This dynamic solution for the free-falling block generates the largest waves in the transient regime, hence the classic steady-state solutions do not really emerge from the above-described solution. Finally, the over-arching assumption is that linear long-wave theory can be used for the generation and local propagation of the waves (see WATTS, 1998, for further discussion of linearity criteria).

\subsection{Evaluation of Landslide Dynamics}

Although an analytical formula has been derived for wave resistance, substitution of this full formulation into the force balance equation produces a highly nonlinear integro-differential equation for block velocity evolution. Here, the force balance of Equation (3.3) is written with full-normalization and substitution of explicit dependencies, except for the wave force term where the summary formula of Equation (3.4) is used:

$$
\begin{aligned}
\partial / \partial t\left(V / C_{w}\right)= & \left.\left\{\Delta \rho / \rho_{a} 1 /\left(W / C_{w}\right)\right)\right\} \\
& \left\{1-F_{\text {frict }} / F_{\text {grav }}-\Delta \rho / \rho D\left(V / C_{w}\right)^{2}-\Delta \rho / \rho b / W \sin \delta\left[A_{>}+A_{<}\right]\right\},
\end{aligned}
$$

where all velocities are normalized by the natural system velocity $C_{w}=(g W \sin \delta)^{1 / 2}$, hence $W / C_{w}$ is the system time scale, and $\rho_{a}$ is the relevant slider density for inertia which includes the added mass coefficient (small for the low aspect slider).

The $A$ functions remain much less than 1 for $V / C$ much less than 1 . If $V / C$ is around 1 , then $A>$ grows as $\approx \Delta d / W$ for displacement $\Delta d$ at $V / C \approx 1$. Thus, basic consideration of force magnitudes in Equation (3.6) demonstrates that the wave resistance term will be small if $V / C$ stays much smaller than 1 during the landslide. If the wave resistance term can be ignored, then the primary force balance is between gravity, inertia, and hydrodynamic drag. The velocity initially increases linearly due to gravitational free-fall down the slope, but as the velocity approaches the limiting value for a particular choice of $D$, the rate decreases. This behavior is similar to that for a purely linear negative feedback which displays an exponential approach to the limiting velocity; there is just a quantitative difference for the (velocity) ${ }^{2}$ feedback. If we make a reasonable choice for the $\Delta \rho / \rho$ density ratio to be 1 , then the limiting nondimensionalized (velocity) ${ }^{2}$ is simply the inverse of the drag coefficient. For a choice 
of $D \approx 1$, this implies that slider velocity cannot exceed the natural system velocity of $C_{w}$.

To now consider the wave resistance force, the key physical parameter is the ratio of slider velocity to wave velocity $V / C$, where $C$ depends on $d$ as $C=(g H(d))^{1 / 2}$, where $H(d)$ is the variable water depth above the slider as it displaces toward deeper water. To make a large wave resistance force, the slider must move with a velocity close to the local wave velocity over a displacement that is a substantial fraction of $W$. If the wave velocity is faster than the limiting block velocity due to drag, then the wave amplitude and wave resistance will remain small. The geologic conclusion for this situation is quite simple: if $H_{o} / W \sin \delta \gg 1 / D$, then the maximum tsunami wave height generated at the source region will always be a small fraction of $b$, the block height.

The above considerations indicate that larger tsunami waves are generated when the initial water depth above the slider $H_{o}$ is much smaller than the characteristic vertical drop of the slider block, $W \sin \delta$ (WATTS, 1998). This basic fact is one of the most important conclusions of this paper; it also conforms to our intuition about wave generation by slides.

We now further investigate a particular case where the block can accelerate until the wave resistance term approaches 1 to balance the gravitational force. Choose the $\Delta \rho / \rho$ and $\Delta \rho / \rho_{a}$ density ratios to be 1 , let the slider geometry ratio $b / W \sin \delta$ be 1 , choose initial water depth to make the $H_{o} / W \sin \delta$ ratio be 1 , and then choose a low drag coefficient of $D=0.1$ so that the $C(d)$ line plots below the limiting drag velocity (see Fig. 4b). For the synthetic landslide generated by these parameter choices, the block displacement, velocity, and wave-making force are plotted as a function of time in Figure 4a. The physical connection between these variables is more easily seen in the "phase space" plot of velocity squared versus displacement (Fig. 4b). For the particular parameter choices made here, $V / C(0)$ reaches an initial peak value of about 1 at displacement $d / W=1$, and the wave force term is about $1 / 2$ which causes velocity to grow slowly until $d / W=2$. This system path is plotted in Figure $4 \mathrm{~b}$. Eventually $V / C(0)$ begins to increase as the slider moves into deeper water, but note that the trajectory slope is less than the wave velocity line (bold line in Fig. 4b), hence the local $V / C(d)$ ratio will never approach 1 in deeper water. Note that somewhere to off the right edge of Figure 4, interface friction increases from zero such that slider velocity will decline back toward zero. The parameter values for the run in Figure 4 were chosen to maximize tsunami generation at a fixed ratio of $H_{o} / W \sin \delta=1$. Thus, other parameter combinations will produce phase space paths that plot in the lower-right section of Figure $4 \mathrm{~b}$ - and hence produce a smaller tsunami.

\subsection{Some Details on Wave Amplitudes and Energy Partitioning}

Let us now turn to some details of the generated tsunami wave heights and associated energy balance. One difference between the steady-state and transient 
solutions is that the transient case sends waves in both the downslope and upslope directions. In fact, for low $V / C$ ratios, the wave heights are comparable, albeit quite small. As $V / C$ approaches 1 , wave heights become more asymmetric with higher waves in the direction of block motion. As seen above, even when choosing parameter values that maximize wave height, the $V / C(d)$ ratio can only
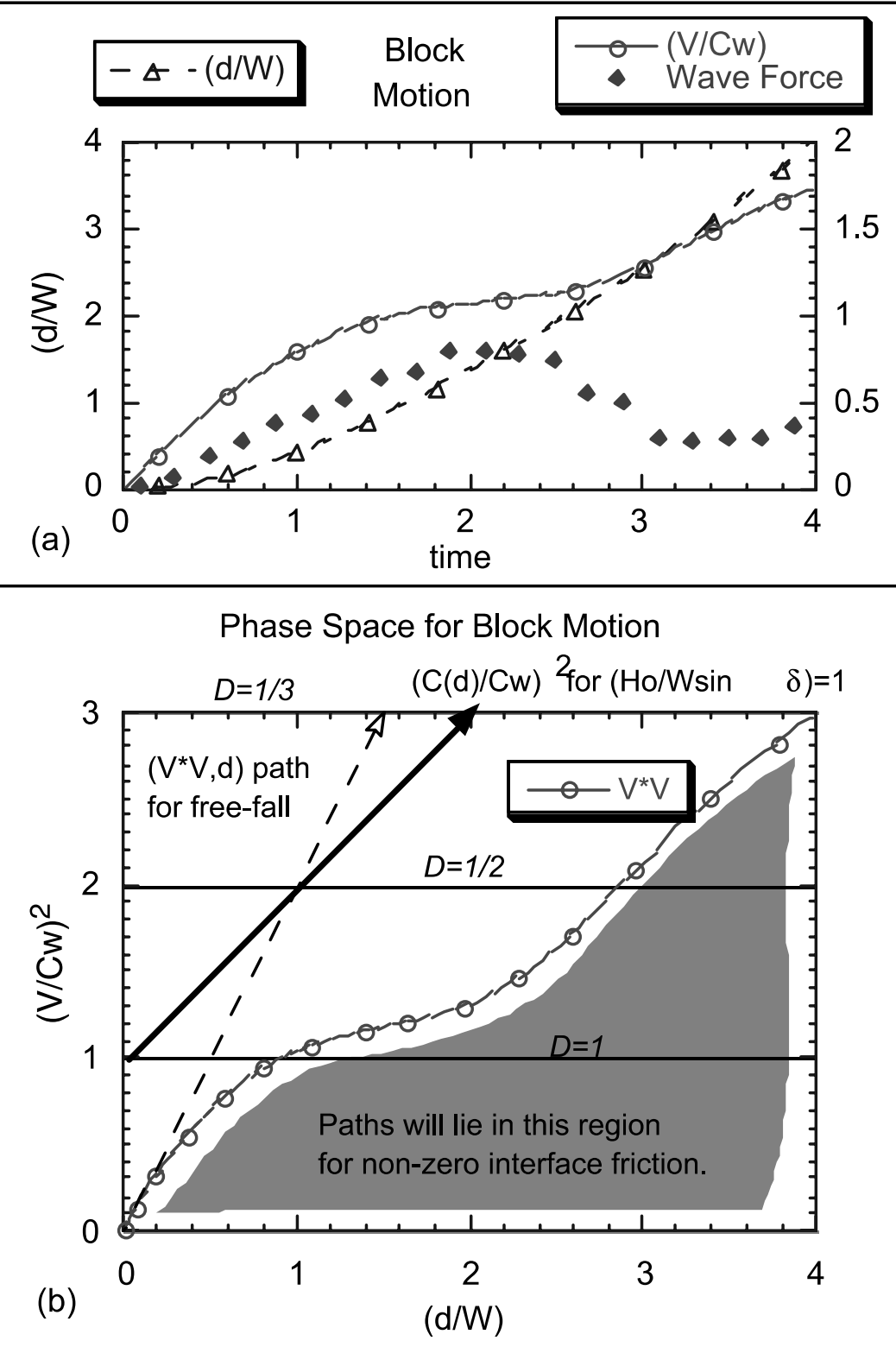
be close to 1 for a small sliding distance (compared to $W$ ). This initial transient wave is "up" in the downslope direction, "down" in the upslope direction (see waves in Figure 5 for the specific case discussed in section 3.3). If the dynamic interface friction can then jump to a very large value (i.e., $F_{\text {frict }} / F_{\text {grav }}>5$ ) to quickly stop the slider while $V / C$ is still close to 1 , then large waves can be produced by this truncation transient - they can be nearly the same amplitude as the initial transients, though of opposite polarity. On the other hand, a gradual deceleration of the slider produces a very long wavelength ramp of very small wave amplitude.

Final energy conversion from gravitational into wave energy depends on the abruptness of motion termination and the total distance traveled beyond the initial $d=2 W$. To generate the largest waves for given values of $D$ and $H_{o} / W \sin \delta$, let dynamic friction drop to zero and let the slider block displace to $d / W=2$, then abruptly truncate the motion so that no energy is lost into friction but instead goes into the large truncation waves. For realistic choices of both $D$ and $H_{o} / W \sin \delta$ equal to 1.0 , then this abrupt truncation behavior can convert about $45 \%$ of the net gravitational energy into wave energy. For even more realistic cases where interface friction slows the block gradually so that $d / W \gg 2$, most of the additional gravitational energy release will be deposited into water drag and interface friction; thus, the wave conversion factor systematically declines with increasing $d$. Even if the initial transient waves have the maximum amplitude, the final value for energy conversion from gravitational into wave energy could be much less than $10 \%$ if the slide continues for great distances. Thus, maximum wave amplitude is a more robust system parameter than final energy conversion efficiency.

\section{Figure 4}

(a) An example synthetic landslide is plotted. The non-dimensionalized block displacement $d / W$, velocity $V / C_{w}$, and wave force are graphed as a function of non-dimensionalized time (see text for definitions). The displacement function uses the left-hand axis, velocity and wave force use the right-hand axis. Parameters for this particular example are chosen to maximize tsunami wave generation; this is verified as the wave force approaches a value of 1 for a brief time. (b) "Phase space" to track the position and velocity of the block during a landslide. It is best to plot (velocity) ${ }^{2}$, non-dimensionalized by $C_{w}^{2}$, versus displacement $d$, non-dimensionalized by $W$. Hydrodynamic drag limits the long-term slider velocity; horizontal lines show velocity bounds for drag coefficient $D$ choices of $1 / 3,1 / 2$, and 1 . Even more important is the $H_{o} / W \sin \delta$ ratio, initial water depth to slider vertical drop, since this ratio sets the vertical offset of wave velocity $C(d)^{2}$ lines (see the bold velocity line plotted for $\left(C(0) / C_{w}\right)^{2}=H_{o} / W \sin \delta=1$ ). The dashed line shows system trajectory for gravitational free-fall with no resistive forces; all system trajectories must fall below this line, though slider paths can initially follow this line for zero dynamic friction. To maximize tsunami wave generation, the hydrodynamic drag line should plot above the wave velocity line so that slider velocity can approach the wave velocity. The system path is plotted (the " $V^{*} V$ " function) for the landslide example shown in (a), where all system ratios are 1 except for $D=0.1$ so that wave force is more important than hydrodynamic drag over the plotted displacement range. 


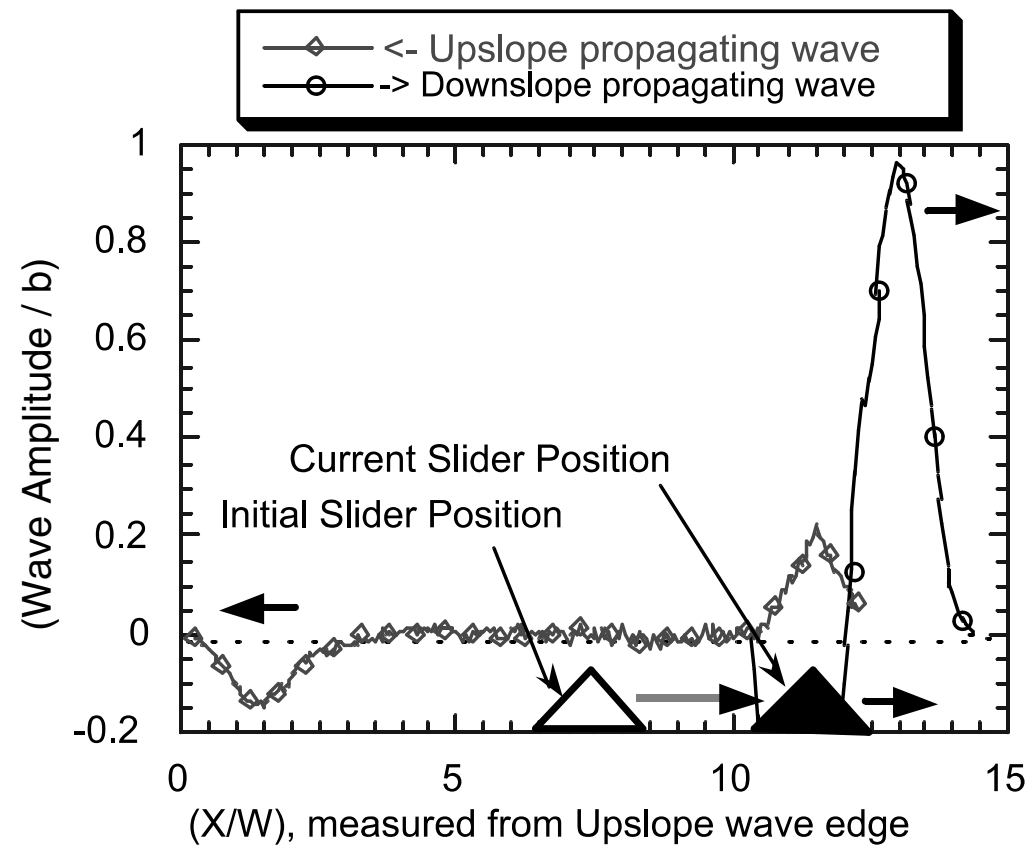

Figure 5

Plots of tsunami waves generated by the system path shown in Figure 4. Horizontal scale measures distance along sea surface, normalized by $W$, and amplitudes are normalized by $b$, slider block height. The choice of small $D$ and $H_{o} / W \sin \delta$ produces nearly maximal wave heights, about +1 in the downslope direction and -0.15 in the upslope direction. Continuation of wave plots over the current position of slider block shows the truncation waves that would propagate away if the block were abruptly stopped. Note the large vertical exaggeration for the triangular block.

\subsection{More Details on Wave Amplitude Systematics}

To focus on the systematic quantitative aspects of these transient waves, the force feedback of waves upon block velocity results in a stable moderation of maximum tsunami wave height. If we specify both $H_{o} / W \sin \delta$ and $D$ ratios to be small, which maximizes tsunami wave height, the tsunami wave amplitude in the downslope direction is never much larger than $b$. This natural buffering effect produces an excellent "rule of thumb": if $H_{o} / W \sin \delta<1$, maximum tsunami wave height at the source region is the same as slider block height, to within $30 \%$ (see Fig. 6 for details). We also find a summary rule for downslope tsunami wave height when the $H_{o} / W$ $\sin \delta$ ratio is larger. Set the system density, geometry, and drag ratios all to 1 , then the ratio of maximum tsunami wave height to $b$, the slider block height, approximately depends on $H_{o} / W \sin \delta$ as follows:

$$
(\max \text { wave height }) / b=\left[1+H_{o} / W \sin \delta\right]^{-1} .
$$

This approximate formula is valid for the $H_{o} / W \sin \delta$ ratio up to about 3 or so, though still valid at the $50 \%$ level for $H_{o} / W \sin \delta$ up to 10 . Figure 6 schematically 


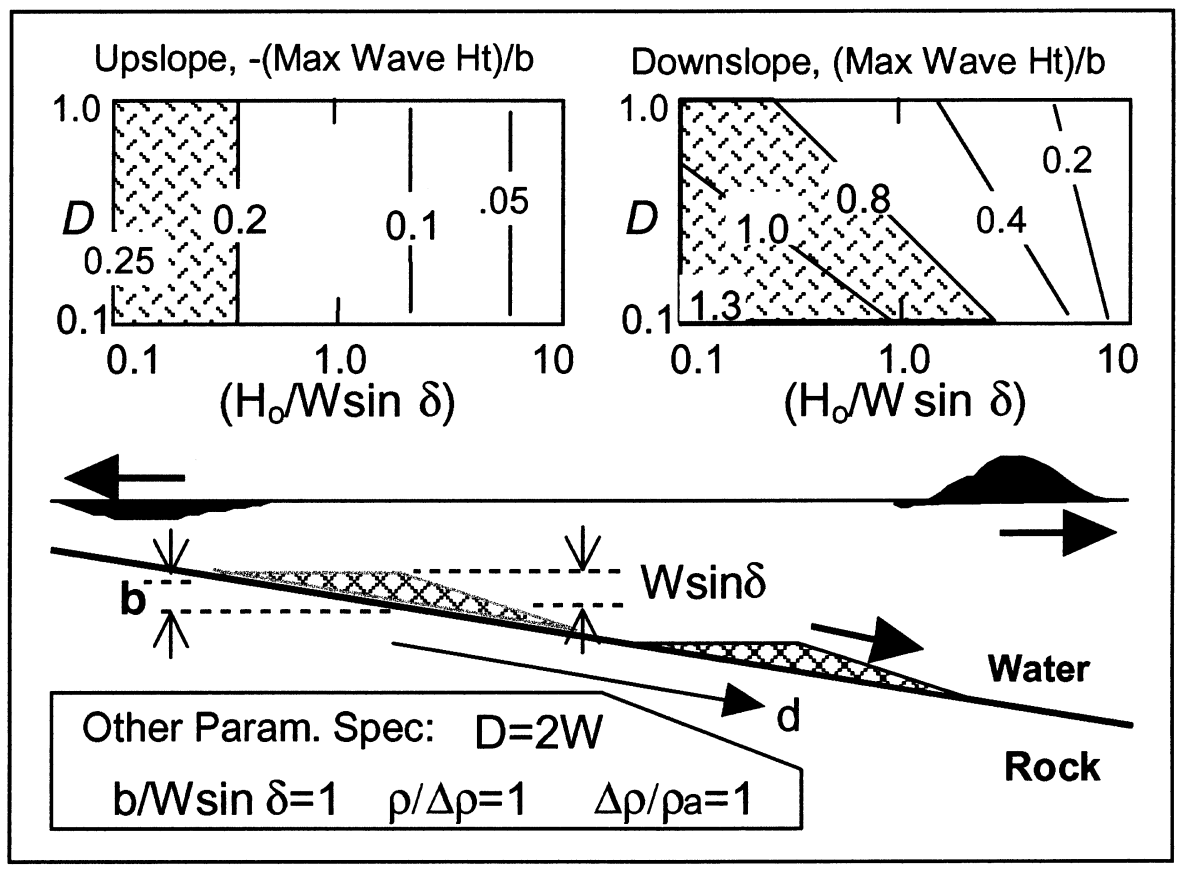

Figure 6

Summary dependence of tsunami wave heights on drag coefficient $D$ and initial water depth $H_{o} / W \sin \delta$. The two graphs above up- and downslope propagating waves show approximate contours of normalized maximum wave heights (multiply by $b$ for actual wave heights). The hachured regions show the realm of the nearly constant largest wave heights. For the downslope/upslope waves, the scale-length from leading edge of wave to peak wave amplitude changes from about $2 \mathrm{~W} / 1.5 \mathrm{~W}$ at $H_{o} / W \sin \delta=10$ to $1 \mathrm{~W}$ in both hachured regions. This figure can be used to provide quick estimates of tsunami wave heights and pulse widths given the basic size, geometry, and water depth of candidate submarine landslides.

graphs the maximum wave amplitudes over variations in both $H_{o} / W \sin \delta$ and $D$. If we perturb the other system ratios, then the maximum wave height in the downslope direction for physically realizable parameter combinations is about twice the block height.

If the landslide occurs in a closed basin, then both downslope and upslope waves can be hazardous. But for many of the geologic settings of triggered landslides, the upslope propagating wave presents the greatest hazards to the nearby coasts. For both the 1992 Flores Island and 1998 PNG events, it was the immediate proximity of the coast to the upslope edge of the slide that caused terrible disasters. Thus, let us also look at the upslope wave amplitudes. The maximum amplitude in the upslope direction is always less than for the downslope direction. For values of $H_{o} / W \sin \delta$ more than 3, the upslope wave amplitude is about half the downslope amplitude. As $H_{o} / W \sin \delta$ becomes quite small, then the upslope amplitude is about one-fourth the downslope amplitude. Thus, we can extract another useful "rule of thumb": if the landslide initiates in very shallow 
water such that the $H_{o} / W \sin \delta$ ratio is less than 1, then the maximum tsunami wave amplitude generated in the source region is approximately $b / 4$, where $b$ is the block height. Of course, this initial source region height of the 2-D wave can be enhanced due to propagation effects near the shore. Figure 6 shows schematic graphs that summarize these amplitude results.

To summarize, the 2-D block dynamic problem produces a self-regulating feedback so that the $V / C$ ratio achieves of value of 1 only for slip distances small compared to $W$, and then only if the initial water depth is less than the vertical drop of the slider. In this regime, tsunami wave heights are "buffered" to be about $b$, slider block height, in the downslope direction, and $b / 4$ in the upslope direction.

\section{Conclusions}

The source theory of tsunami waves generated by earthquakes is a wellestablished procedure and the energy balance results derived here support the basic kinematic methodology by virtue of the fact that maximum tsunami wave energy will always be less than $1 \%$ of the minimum earthquake energy. This small energy conversion factor from earthquakes into tsunamis is still responsible for most of the destructive tsunamis in the world.

Marine surveys have now documented ancient submarine landslides that rival the greatest earthquakes in their total energy release. The expected annual occurrence of huge submarine landslides is still an unanswered question, but even small submarine landslides can cause large localized tsunamis, as evidenced by recent earthquake-triggered slides. My quantitative exploration of tsunami wave source theory from submarine landslides shows that gravitational to wave energy conversion can range from essentially zero to about $50 \%$ as a practical upper bound for reasonable geologic and hydrodynamic parameters. This wide range in energy conversion produces a highly variable efficiency in tsunami generation by submarine landslides. The key parameter is the ratio of initial water depth above the block-to-block vertical scale height; this ratio must be less than 1 for the landslide to generate large waves. Indeed, for future detailed numerical modeling, a glance at Figure 6 will already forecast whether significant tsunami waves can be generated for a particular physical setting. Turning to the practical key parameter of maximum tsunami wave height in the source area, the interaction of all the dynamic effects produces a remarkably stable and simple result: if the key ratio of water depth to block vertical scale height is small such that a significant tsunami will be generated, then the maximum tsunami height will be approximately equal to the block height - largely independent of the other parameters! The graphs in Figure 6 give more detailed information on maximum wave amplitudes for both the downslope and upslope propagating waves. Figure 6 provides a simple yet useful evaluation of tsunami hazards for submarine landslides. 


\section{Acknowledgments}

Thanks to the organizers and participants in the "Workshop on the Prediction of Underwater Landslide and Slump Occurrence and Tsunami Hazards off of Southern California," held in Los Angeles, CA in March, 2000. This work was presented there, and I enjoyed the scientific discussions and feedback at this gathering of the world's tsunami community. I also appreciate the many discussions on earthquakes, tsunamis, and landslides over the years with my Michigan colleagues - especially Kenji Satake and Yuichiro Tanioka. Special thanks to Phil Watts for his encouragement and constructive comments. The research program on earthquakes, tsunamis, and related phenomenon over the years at the University of Michigan has been supported by the National Science Foundation (EAR-9725175 and OCE9905503) and USGS (GR-02754).

\section{REFERENCES}

AвE, K. (1979), Size of Great Earthquakes of 1873-1974 Inferred from Tsunami Data, J. Geophys. Res. 84, $1561-1568$.

Dahlen, F. (1977), The Balance of Energy in Earthquake Faulting, Geophys. J. Roy. Astron. Soc. 48, 239261.

Grilli, S. and Watts, P. (1999), Modeling of Waves Generated by a Moving Submerged Body: Applications to Underwater Landslides, Engin. Analysis Boundary Elements 23, 645-656.

Hampton, M. A., Lee, H., and Locat, J. (1996), Submarine Landslides, Rev. Geophys. 34, 33-59.

Hasegawa, H. and Kanamori, H. (1987), Source Mechanism of the Magnitude 7.2 Grand Banks Earthquake of November 1929: Double Couple or Submarine Landslide? Bull. Seismol. Soc. Am. 77, 1984 2004.

Haskell, N. (1964), Total Energy and Energy Spectral Density of Elastic Wave Radiation from Propagating Faults, Bull. Seismol. Soc. Am. 54, 1811-1842.

Hidayat, D., Barker, J., and Satake, K. (1995), Modeling the Seismic Source and Tsunami Generation of the December 12, 1992 Flores Island, Indonesia Earthquake, Pure Appl. Geophys. 144, 537-554.

Imamura, F., Gica, E., Takahashi, T., and Suto, N. (1995), Numerical Simulation of the 1992 Flores Tsunami: Interpretation of Tsunami Phenomenon in Northeastern Flores Island and Damage at Babi Island, Pure Appl. Geophys. 144, 555-568.

Iwasaki, S., Furumoto, A., and Honza, E. (1996), Can a Submarine Landslide be Considered as a Tsunami Source? Tsunami Hazards 14(2), 89-100.

Johnson, C. and Mader, C. (1994), Modeling of the 105 Ka Lanai Tsunami, Tsunami Hazards 12(1), 3338.

Kanamori, H. (1972), Mechanisms of Tsunami Earthquakes, Phys. Earth Planet. Int. 6(5), 346-359.

Kanamori, H. (1977), The Energy Release in Great Earthquakes, J. Geophys. Res. 82, 2981-2987.

Kawata, Y., Benson, B., Borerro, J., Borerro, J. L., Davies, H., delange, W., Imamura, F., Letz, H., Nott, J., and Synolakis, C. E. (1999), Tsunami in Papua New Guinea was as Intense as First Thought, EOS, Trans. AGU 80, 101-105.

KIKUChI, M. and FukAo, Y. (1988), Seismic Wave Energy Inferred from Long-period Body Wave Inversion, Bull. Seismol. Soc. Am. 78, 1707-1724.

Lamb, H. (1932), Hydrodynamics (Cambridge Univ. Press) 6th ed., 738.

Lighthill, J. (1978), Waves in Fluids (Cambridge Univ. Press) 504.

Ma, K., Kanamori, H., and Satake, K. (1999), Mechanism of the 1975 Kalapana Hawaii Earthquake Inferred from Tsunami Data, J. Geophys. Res. 104, 13,153-13,167. 
Miller, D. J. (1960), The Alaska Earthquake of July 10, 1958: Giant Wave in Lituya Bay, Bull. Seismol. Soc. Am. 50, 252-266.

Moore, J., Normark, W., and Holcomb, R. (1994), Giant Hawaiian Landslides, Annu. Rev. Earth Planet. Sci. 22, 119-144.

Murty, T. S. (2003), Tsunami Generation from Submarine Landslides, Pure Appl. Geophys. 160, 21472153.

Nomanbhoy, N. and Satake, K. (1995), Generation Mechanism of Tsunamis from the 1883 Krakatau Eruption, Geophys. Res. Lett. 22, 509-512.

Okal, E. (1988), Seismic Parameters Controlling Far-field Tsunami Amplitudes: A Review, Natural Hazards 1, 67-96.

Piper, D., Shor, A., Farre, J., O’Connell, S., and Jacobi, R. (1985), Sediment Slides and Turbidity Currents on the Laurentian Fan: Sidescan Sonar Investigations near the Epicenter of the 1929 Grand Banks Earthquake, Geology 13, 538-541.

Ruff, L. (1999), Dynamic Stress Drop of Recent Earthquakes: Variations within Subduction Zones, Pure Appl. Geophys. 154, 409-431.

Satake, K. (1995), Linear and Non-linear Computations of the 1992 Nicaragua Earthquake Tsunami, Pure Appl. Geophys. 144, 455-470.

Sweet, S. and Silver, E. A. (2003), Seismic Reflection Images of the Source Region of the 1998 Papua New Guinea Tsunami, Pure Appl. Geophys. 160, 1945-1968.

Synolakis, C. and Tadepalli, S. (1993), The Runup of Dipole Waves, Proc. IUGG/IOC Inter. Tsunami Symp. 175-187.

TANiOKA, Y. (1999), Analysis of the Far-field Tsunamis Generated by the 1998 Papua New Guinea Earthquake, Geophys. Res. Lett. 26, 3393-3396.

Tappin, D., Watts, P., McMurty, G., Lafoy, Y., and Masumoto, T. (2003), Prediction of Slump Generated Tsunamis: The July 17, 1998 Papua New Guinea Tsunami.

TichelaAr, B. and Ruff, L. (1993), Depth of Seismic Coupling along Subduction Zones, J. Geophys. Res. 98, 2,017-2,037.

Titov, V. and Synolakis, C. E. (1993), A Numerical Study of Wave Runup of the September 2, 1992, Nicaraguan Tsunami, Proc. IUGG/IOC Inter. Tsunami Symp., 627-635.

Vassiliou, M. S. and Kanamori, H. (1982), The Energy Release in Earthquakes, Bull. Seismol. Soc. Am. $71(2), 371-387$.

WArd, S. N., Tsunamis. In Encyclopedia of Physical Science and Technology (Academic Press, New York: 2000).

Watts, P. (1998), Wavemaker Curves for Tsunamis Generated by Underwater Landslides, J. Waterway Port Coastal Ocean Eng. 124(3), 127-137.

Watts, P. and Borrero, J. C. (2003), Probabilistic Predictions of Underwater Landslides and Tsunami Amplitudes

Winslow, N. W. and Ruff, L. J. (1999), A Hybrid Method for Calculating the Radiated Wave Energy of Deep Earthquakes, Phys. Earth Planet. Int. 115, 181-190. (20) To access this journal online: 